\title{
Virtual prediction of antiviral potential of ginger (Zingiber officinale) bioac- tive compounds against spike and MPro of SARS-CoV2 protein
}

\author{
Ahmad Hafidul Ahkam ${ }^{1}$, Feri Eko Hermanto ${ }^{1}$, Adzral Alamsyah ${ }^{1}$, Iva Himmatul Aliyyah ${ }^{1,2}$, Fatchiyah Fatchiyah ${ }^{1,2,}$ \\ ${ }^{1}$ Department of Biology, Faculty Mathematics and Natural Sciences, Universitas Brawijaya, Malang, Indonesia \\ ${ }^{2}$ Research Center of Smart Molecule of Natural Genetics Resources, Universitas Brawijaya, Malang, Indonesia
}

\begin{abstract}
Coronavirus disease 2019 (COVID-19) is a human disease caused by SARS-CoV2 becomes a serious health threat after infected more than 6 million people globally. The virus enters the host cell through an S protein on its surface and begins its life cycle with the help of a key protein, MPro. On the other hand, several bioactive from Ginger have been reported for their antiviral properties, but few studies related to COVID-19. This study aims to pursue the potential of a few bioactive compounds from Ginger as anti-SARS-CoV 2 from their interaction to spike and Mpro protein. Gingerenone A, gingerol, geraniol, shogaol, zingiberene, zingiberenol, and zingerone were used as ligand to be docked with S protein and MPro. Drug-likeness properties also evaluated using SwissADME. Gingerenone A constantly gave the lowest binding energy compared to others both with S or MPro. However, gingerol, geraniol, shogaol, zingiberene, zingiberenol, and zingerone could interact with key residues responsible for MPro catalytic domain, while geraniol, shogaol, zingiberene, zingiberenol, and zingerone could interfere S-ACE2 binding shape and increase its binding energy. The drug-likeness analysis also revealed that all of the analyzed compounds have no violation of Lipinski's Rule of 5. In conclusion, gingerol, geraniol, shogaol, zingiberene, zingiberenol, and zingerone from Ginger have good potential as antiviral agents with good oral bioavailability and flexibility.
\end{abstract}

Keywords: COVID-19, Ginger, MPro, SARS-COV 2, Spike protein

Received: 8 June 2020 Revised: 13 June 2020 Accepted: 16 June 2020

\section{Introduction}

The world is facing one major health threat, namely COVID-19. The pandemic was caused by the SARSCoV2 virus which was first identified in Wuhan, China (Wang et al., 2020). The number of positive cases continues to grow every day, reaching more than 6 million infected people spread in more than 200 countries (https://covid19.who.int/). Indonesia has a total of 27,549 cases (update June 6, 2020) and is predicted to grow over time (https://covid19.go.id/). With this crisis, there is an urgent to develop an agent to drive the COVID-19 outbreak.

The Spike (S) protein is one of SARS-CoV2's protein responsible for viral entry during the infection process (Walls et al., 2020). It will bind with AngiotensinConverting Enzyme 2 (ACE2) receptor from the host cell to create a suitable habitation for viral replication (Walls et al., 2020). Due to its important role, several drug development used $\mathrm{S}$ protein as a target to prevent the disease (Tu et al., 2020; Xia et al., 2020). Main Protease (MPro) is also one of the essential enzymes during viral replication. This protein is accountable for processing the poly-proteins pp1a and pplab into established nonstructural protein as a part of the replication/transcription

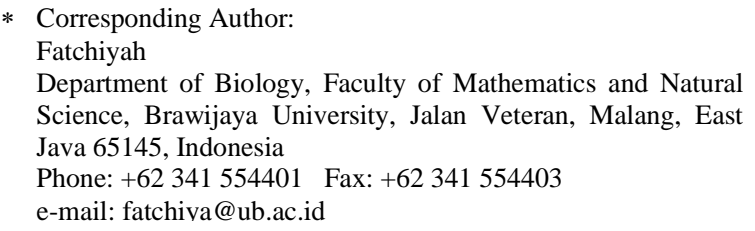

complex (Hilgenfeld, 2014). Thus, targeting MPro also promising to combat COVID-19 (Bzówka et al., 2020).

Natural-derived compounds constantly become a worthy therapeutic alternative against several diseases, including viral infection. Herbal exploration continually performed, also in order to diminish coronavirus-related disease (Islam et al., 2020; Li et al., 2005). Ginger (Zingiber officinale) as a well-known herbal plant has a proper candidate because it has a lot of biologically active compounds. It has been shown to provide antioxidant, anti-inflammatory, anticancer, and antimicrobial activities, and the potential as antiviral component (Mao et al., 2019; Rasool et al., 2017). Therefore, this study was designed to search for the possibility of antiviral properties of Ginger in counteracting SARS-CoV 2 infection base on ligand interaction to spike and MPro protein. In silico test was carried out on active compounds of Ginger plants by docking on $\mathrm{S}$ protein and MPro. Therefore, it is expected that several active compounds from Ginger are able to block the $\mathrm{S}$ protein from binding to the ACE2 receptor or perform as an inhibitor for MPro.

\section{Methods}

\section{Data Mining/Ligand and Protein Sampling}

The crystallized structures of Spike (S) and ACE2 protein with protein data bank identifier (PDB ID) 6M0J and MPro Protein (PDB ID: 6LU7) were taken from RSCB PDB (https://www.rcsb.org/). Seven active compounds from Ginger which used as ligands were retrieved from ChemSpider with certain ChemSpider 
identifier (CSID) and also their abbreviation, i.e. Gingerenone A (GGR, CSID: 4445088), Gingerol (GGO, CSID: 391126), Geraniol (GRO, CSID: 13849-989), Shogaol (SGO, CSID: 4445106), Zingiberene (ZBR, CSID: 83751), Zingiberenol (ZBO, CSID: 30771649), Zingerone (ZGR, CSID: 28952), and Remdesivir (RDV, CSID: 58827832). Protein structures were downloaded in .pdb format, while ligands were downloaded as .mol format.

Ligand Selection and Drug Likeness Characterization Seven active compounds from Ginger were chosen based on KNApSAcK database (http://www.knapsackfamily.com/). Drug likeness was defined based on SwissADME physicochemical properties, including molecular weight, LogP value, and number of $\mathrm{H}$-bond donor, $\mathrm{H}$-bond acceptor, rotatable bond, and Total Polar Surface Area (TPSA) (Daina et al., 2017).

\section{Molecular Docking}

Proteins were prepared using Discovery Studio version 16 (Dassault Systèmes BIOVIA, 2015) to remove previous attached ligand, while ligands were prepared using Open Babel integrated in PyRx 8.0 (Dallakyan \& Olson, 2015) to minimize its energy and convert it into .pdb format. Molecular docking simulation was performed using Hex software (Macindoe et al., 2010) with Shape+DARS for correlation type and other parameters remain default.

\section{Virtual prediction analysis and visualization}

Protein-ligand complexes from docking step were analyzed and visualized using Discovery Studio. Site of interaction was analyzed based on ligand-residue interaction and structure conformation. RDV also docked with MPro to compare the binding affinity of seven active compounds used in this study according to previous research (Cheng \& Li, 2020).

\section{Results}

\section{Drug Likeness Properties}

All of analyzed compounds have good potential as drug candidates, given the suitability with Lipinski's rule of 5 (LRO5) (Tab. 1). There were no unsuitability in terms of molecular weight, $\log \mathrm{P}$ value, number of $\mathrm{H}$ bond donor and acceptor, number of rotatable bond, and TPSA value. These properties explained that seven active compounds from Ginger have good permeability and flexibility as drug candidates to perform their biological activities.

\section{Ligand Potency as Inhibitor of MPro}

The docking results between seven active Ginger compounds were shown in Table 2. RDV seems to have the lowest energy compared to analyze compounds. GGR has the lowest binding energy compared to six other compounds, but still higher than RDV. Figure 1 showed the differences in the interacting site of each compound. Of the 6 active compounds include GGO, GRO, SGO, $\mathrm{ZBR}, \mathrm{ZBO}$, and ZGR appear to interact in a similar site. However, only GGR appears to interact on different sites compared to other compounds.

Although GGR has the closest binding energy with RDV, both ligands did not interact similarly. None of the interacted residues in MPro-GGR and MPro-RDV has shared in the same residues (Tab. 3). Nevertheless, some amino acids were shared in GGO, GRO, SGO, ZBR, ZBO, and ZGR complexes with MPro, especially His 164 (Tab. 3). Nonetheless, further studies required confirming the potency of GGO, GRO, SGO, ZBR, ZBO, and ZGR in inhibiting MPro.

Table 1. The potential value of Ginger active compounds as medicinal substance

\begin{tabular}{lccccccc}
\hline & GGR & GGO & GRO & SGO & ZBR & ZBO & ZGR \\
\hline Mol. weight (g/mol) & 356.41 & 294.39 & 154.25 & 276.37 & 204.35 & 222.37 & 196.24 \\
Log P & 3.65 & 3.13 & 2.74 & 3.76 & 4.46 & 3.78 \\
H-bond donor & 2 & 2 & 1 & 1 & 0 & 1.86 & 1 \\
H-bond acceptor & 5 & 4 & 1 & 3 & 0 & 1 & 4 \\
Rotatable bonds & 9 & 10 & 4 & 9 & 4 & 4 \\
TPSA & $75.99 \AA^{2}$ & $66.76 \AA^{2}$ & $20.23 \AA^{2}$ & $46.53 \AA^{2}$ & $0.00 \AA^{2}$ & $20.23 \AA^{2}$ & $46.53 \AA^{2}$ \\
\hline
\end{tabular}
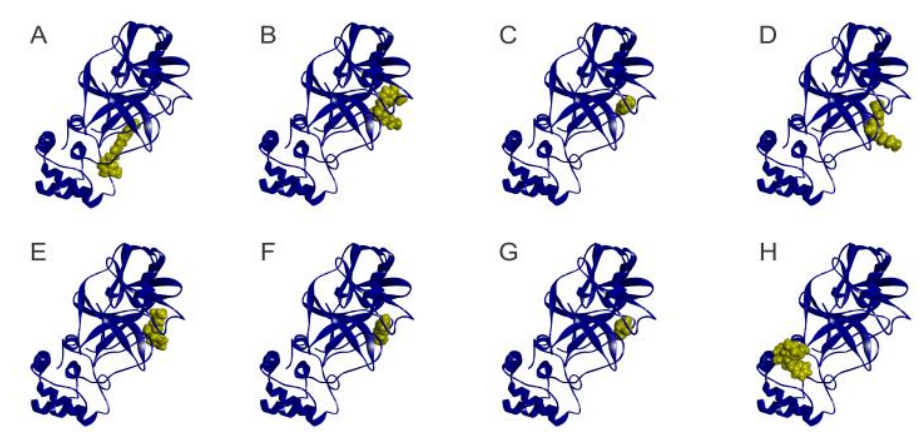

Figure 1. Visualization of MPro complexes with bioactive compounds from Ginger or RDV. Yellow spheres points the interaction site of each ligand in MPro. Blue ribbons denote the proteins, while yellow spheres are the ligands. In an ordered manner, alphabet A-H represent GGR, GGO, GRO, SGO, ZBR, ZBO, ZGR, and RDV, respectively. 


\section{Ligand Potency as Inhibitor of Spike Protein-ACE2 Receptor Complex}

Similar to MPro binding energy results, GGR also showed the lowest binding energy compared to the other six compounds (Tab. 2). Although this energy relatively higher than the S-ACE2 complex, several compounds could reduce the ability of $S$ protein to interact with ACE2 after bounded with the compounds. Those compounds are GRO, SGO, ZBR, ZBO, and ZGR. The energy required for $S$ protein to interact with ACE2 was higher after bounded with GRO, SGO, ZBR, ZBO, or ZGR compared to S-ACE2 complex without ligand (Tab. 2 ). This result suggested that GRO, SGO, ZBR, ZBO, and ZGR have a good potency to prevent viral entry during SARS-CoV2 infection.

Complex structure visualization revealed that every ligand performs a similar binding site, except for ZBR (Fig. 2E). From the interacted residues, ZBR also bound with different amino acids compared to other compounds (Tab. 3). To understand the effect of GRO, SGO, ZBR, $\mathrm{ZBO}$, and ZGR to prevent S-ACE2 interaction, we also executed a structural comparison of S-ACE2 complex with and without ligand bounded with $S$ protein. After $S$ protein docked with GRO, SGO, ZBR, ZBO, or ZGR, the formed complexes then docked again with ACE2. Figure $3 \mathrm{~A}$ and $3 \mathrm{G}$ represent the initial form of S-ACE2 complex without the presence of ligand at $0^{\circ}$ and $180^{\circ}$ angle, respectively. After $\mathrm{S}$ protein bounded with GRO, SGO, $\mathrm{ZBR}, \mathrm{ZBO}$, or ZGR, the structure orientation either $\mathrm{S}$ protein or ACE2 shifted into another way (Fig. 3 B, C, D, $\mathrm{E}, \mathrm{F}, \mathrm{H}, \mathrm{I}, \mathrm{J}, \mathrm{K}, \mathrm{L})$. The detail about interacted residues can be seen in supplementary table 1 . These results revealed that GRO, SGO, ZBR, ZBO, or ZGR able to inhibit S-ACE2 complex formation during viral infection. Advanced studies still needed to confirm those mechanisms.

Table 2. Binding energy of analyzed ligands interacted with targeted proteins

\begin{tabular}{lccc}
\hline \multirow{2}{*}{ Ligand } & \multicolumn{3}{c}{ Binding Energy $(\mathbf{k c a l} / \mathbf{m o l})$} \\
\cline { 2 - 4 } & Mpro & S & S-Ligand-ACE2 \\
\hline GGR & -301.2 & -289.9 & -747.4 \\
GGO & -282.9 & -282.9 & -767.0 \\
GRO & -192.7 & -177.0 & -714.3 \\
SGO & -257.0 & -250.8 & -731.2 \\
ZBR & -234.5 & -218.4 & -714.9 \\
ZBO & -227.1 & -204.5 & -699.2 \\
ZGR & -208.1 & -191.9 & -684.6 \\
RDV & -340.2 & - & - \\
ACE2 & - & -745.4 & - \\
\hline
\end{tabular}
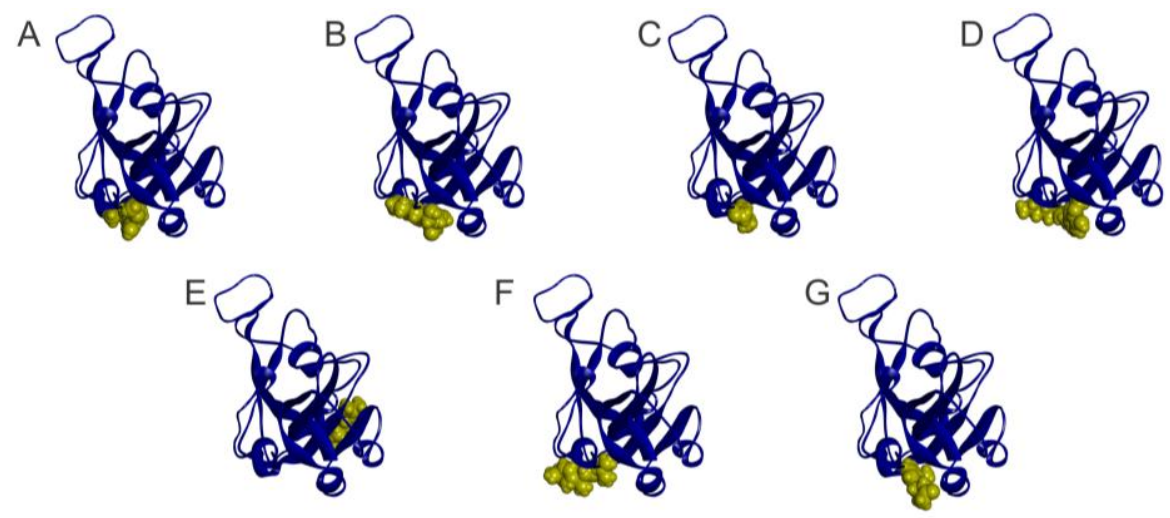

Figure 2. Visualization of S protein complexes with seven bioactive compounds from Ginger. Yellow spheres points the interaction site of each ligand in S protein. Blue ribbons denote the proteins, while yellow spheres are the ligands. In an ordered manner, alphabet A-G represent GGR, GGO, GRO, SGO, ZBR, ZBO, and ZGR, respectively
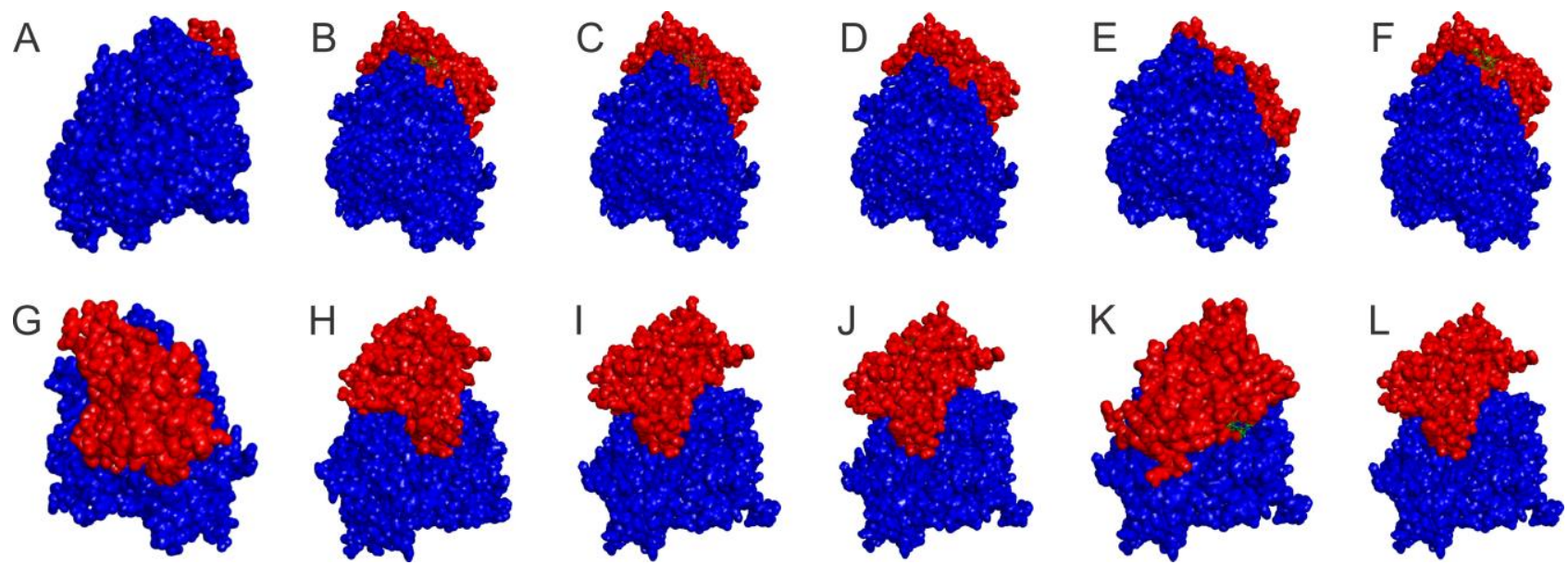

Figure 3. Structural orientation of S-ACE2 (A,G), S-GRO-ACE2 (B,H), S-SGO-ACE2 (C,I), S-ZBR-ACE2 (D,J), S-ZBO-ACE2 (E,K), and S-ZGRACE2 (F,L). Alphabet A-F showed structural orientation at $0^{\circ}$ angle, while G-L at $180^{\circ}$ angle. Red shape and blue shape represent $S$ protein and ACE2, correspondingly. 
Table 3. Interacting residues in MPro and S Protein with ginger bioactive compound

\begin{tabular}{|c|c|c|c|c|c|c|c|c|c|c|c|c|c|c|c|c|}
\hline \multirow{3}{*}{$\begin{array}{l}\text { Protein } \\
\text { MPro }\end{array}$} & \multicolumn{16}{|c|}{ (Type of Interaction) Interacting Residues } \\
\hline & \multicolumn{2}{|l|}{ GGR } & \multirow{2}{*}{\multicolumn{2}{|c|}{ GGO }} & \multicolumn{2}{|l|}{ GRO } & \multicolumn{2}{|c|}{ SGO } & \multicolumn{2}{|c|}{ ZBR } & \multicolumn{2}{|l|}{ ZBO } & \multicolumn{2}{|l|}{ ZGR } & \multicolumn{2}{|l|}{ RDV } \\
\hline & $(\mathrm{VW})$ & Lys 5 & & Leu 27 & $(\mathrm{VW})$ & Thr 25 & (HP) & His 41 & $(\mathrm{VW})$ & Thr 25 & (HP) & His 41 & (VW) & His 41 & (UF) & Gln 107 \\
\hline & (HP) & Tyr 126 & (VW) & His 41 & (HB) & Thr 26 & (HP) & Met 49 & (VW) & Thr 26 & (HP) & Met 49 & (VW) & Met 49 & (ES) & Pro 108 \\
\hline & (VW) & Gln 127 & (VW) & Tyr 54 & (VW) & Leu 27 & (VW) & Tyr 54 & (HP) & Leu 27 & (VW) & Phe 140 & (VW) & Phe 140 & (HB) & Gly 109 \\
\hline & (VW) & Cys 128 & (VW) & Phe 140 & (HP) & His 41 & (VW) & Cys 145 & (VW) & His 41 & (VW) & Leu 141 & (HB) & Leu 141 & (ES) & Gln 110 \\
\hline & (VW) & Arg 131 & (VW) & Leu 141 & (VW) & Phe 140 & (VW) & His 164 & (VW) & Met 49 & (VW) & Asn 142 & (VW) & Asn 142 & (ES) & Pro 132 \\
\hline & (HP) & Lys 137 & (VW) & Asn 142 & (HB) & Leu 141 & (HP) & Met 165 & (VW) & Phe 140 & (VW) & Gly 143 & (VW) & Gly 143 & (HB) & Phe 143 \\
\hline & (VW) & Gly 138 & (VW) & Gly 143 & (VW) & Asn 142 & (VW) & Glu 166 & (HP) & Leu 141 & (VW) & Ser 144 & (UF) & Ser 144 & (HB) & Thr 198 \\
\hline & (VW) & Ser 139 & (VW) & Ser 144 & (VW) & Gly 43 & (VW) & Leu 167 & (VW) & Asn 142 & (HP) & Cys 145 & (HB) & Cys 145 & (HB) & Ile 200 \\
\hline & (VW) & Thr 199 & (HP) & Cys 145 & (VW) & Ser 144 & (HP) & Pro 168 & (VW) & Gly 143 & (HB) & His 163 & (HB) & His 163 & (ES) & Val 202 \\
\hline & (HB) & Tyr 239 & (VW) & His 164 & (HP) & His 163 & (VW) & Arg 188 & (HP) & Cys 145 & (VW) & Met 165 & (VW) & Met 165 & (ES) & Glu 240 \\
\hline & (HP) & Leu 286 & (HB) & Met 165 & (HB) & His 164 & (VW) & Gln 189 & (HP) & His 163 & (VW) & Glu 166 & (VW) & Glu 166 & (HB) & Pro 241 \\
\hline & (VW) & Leu 287 & (HB) & Glu 166 & (VW) & Met 165 & (VW) & Thr 190 & (VW) & His 164 & (HP) & His 172 & (VW) & His 172 & (HB) & Thr 243 \\
\hline & (VW) & Glu 288 & (VW) & His 172 & (VW) & Glu 166 & (VW) & Ala 191 & (VW) & Met 165 & (VW) & $\operatorname{Arg} 188$ & (VW) & Asp 187 & (HB) & Asp 245 \\
\hline & (VW) & Asp 289 & & & (HB) & His 172 & (VW) & Gln 192 & (VW) & Glu 166 & (VW) & Gln 189 & (VW) & Arg 188 & (VW) & His 246 \\
\hline & (VW) & Glu 290 & & & & & & & (VW) & His 172 & & & (VW) & Gln 189 & (ES) & Ile 249 \\
\hline & & & & & & & & & & & & & & & (HB) & Thr 292 \\
\hline & & & & & & & & & & & & & & & (ES) & Phe 294 \\
\hline & & & & & & & & & & & & & & & (ES) & Pro 298 \\
\hline \multirow[t]{15}{*}{ Spike } & (HP) & Leu 335 & (VW) & Leu 335 & (VW) & Cys 336 & (VW) & Phe 338 & (VW) & Ser 375 & (HP) & Phe 342 & (VW) & Gly 339 & - & - \\
\hline & (HP) & Cys 336 & (UF) & Cys 336 & (VW) & Phe 338 & (HP) & Phe 342 & (UF) & Thr 376 & (VW) & Asn 343 & (HB) & Phe 342 & & \\
\hline & (HP) & Phe 342 & (VW) & Gly 339 & (VW) & Asn 343 & & & & & (VW) & Phe 347 & (VW) & Val 367 & & \\
\hline & (VW) & Asn 343 & (VW) & Glu 340 & (VW) & Asn 343 & (VW) & Thr 345 & (VW) & Asp 405 & (VW) & Ser 373 & (VW) & Leu 368 & & \\
\hline & (VW) & Ala 363 & (VW) & Phe 342 & (VW) & Val 367 & (HB) & Val 367 & (HP) & Val 407 & (HP) & Phe 374 & (VW) & Ser 371 & & \\
\hline & (HB) & Asp 364 & (VW) & Asn 343 & (HP) & Leu 368 & (HP) & Leu 368 & (HP) & Arg 408 & (HP) & Trp 436 & (HB) & Ser 373 & & \\
\hline & (HB) & Val 367 & (VW) & Val 362 & (VW) & Ser 371 & (VW) & Ser 371 & (VW) & Gln 409 & (HP) & Leu 441 & (HP) & $\operatorname{Trp} 436$ & & \\
\hline & (HP) & Leu 368 & (VW) & Ala 363 & (VW) & Ser 373 & (VW) & Ser 373 & (VW) & Ile 410 & (VW) & Arg 509 & & & & \\
\hline & (UF) & Leu 368 & (UF) & Asp 364 & (HP) & Phe 374 & (HP) & Phe 374 & (HP) & Ala 411 & & & & & & \\
\hline & (VW) & Ser 371 & (VW) & Val 367 & (HP) & Trp 436 & (HP) & Trp 436 & (VW) & $\operatorname{Gln} 414$ & & & & & & \\
\hline & $(\mathrm{VW})$ & Ser 373 & (HP) & Leu 368 & & & (VW) & Asn 440 & (VW) & Val 433 & & & & & & \\
\hline & $\begin{array}{l}(\mathrm{VW}) \\
\text { (HP) }\end{array}$ & $\begin{array}{l}\text { Phe } 374 \\
\text { Trp } 436\end{array}$ & $\begin{array}{l}(\mathrm{VW}) \\
(\mathrm{VW})\end{array}$ & $\begin{array}{l}\text { Ser } 371 \\
\text { Ser } 373\end{array}$ & & & $\begin{array}{l}\text { (VW) } \\
\text { (HP) }\end{array}$ & $\begin{array}{l}\text { Asn } 440 \\
\text { Leu } 441\end{array}$ & (VW) & Ala 435 & & & & & & \\
\hline & (HP) & Leu 441 & (VW) & Phe 374 & & & (VW) & $\operatorname{Arg} 509$ & (HP) & Val 503 & & & & & & \\
\hline & & & & & & & & & (VW) & Gly 504 & & & & & & \\
\hline & & & & & & & & & (VW) & Tyr 508 & & & & & & \\
\hline
\end{tabular}

Note: $\mathrm{ES}$ = Electrostatic; HB = Hydrogen Bond; HP = Hydrophobic; UF = Unfavourable bond; VW = Van der Waals 


\section{Discussion}

Physicochemical properties strongly correlated with drug-likeness of a compound to perform a certain biological activity and it was properly guided with LRO5. Compound with no violation of LRO5 will give good oral bioavailability and fit drug absorption, distribution, efficacy, metabolism, and excretion (ADME) regulation (Lipinski, 2004). Referring to LRO5, a compound will adequate to drug-like molecule if it has a molecular weight less than $500 \mathrm{~g} / \mathrm{mol}$, a $\log \mathrm{P}$ value no more than 5 which represents hydrophobicity characters, number of $\mathrm{H}$ bond donor less than 5, and H-bond acceptor less than 10 sites. Further, the number of rotatable bonds less than 10 will provide molecule flexibility, while TPSA value less than or equal to $140 \AA$ provides good permeability and bioavailability (Chagas et al., 2018). Regarding those rules, all of the analyzed compounds from Ginger have acceptable to perform respectable drug-likeness properties.

Several drugs used the MPro enzyme as a target to inhibit polyprotein processing during viral RNA translation (Bzówka et al., 2020; Dai et al., 2020; Joshi et al., 2020; Zhang et al., 2020). MPro of SARS-CoV2 has three domains with an active site in the middle of domain I and domain II. It has a close similarity to SARS-CoV's MPro with catalytic residues located in His 41-Cys 145 (Gurung et al., 2020). Among several residues in those active sites, Cys 145 could be bounded by GGO, GRO, SGO, ZBR, ZBO, and ZGR (Tab. 3). However, RDV didn't appear to be bounded to the active site of MPro, suggesting that active compounds from Ginger have better potency as an MPro inhibitor to prevent its catalytic activity.

$\mathrm{S}$ protein is the main mediator of SARS-CoV2 to interact with the host's ACE2 so it can enter the host cells (Walls et al., 2020). A region called Receptor Binding Domain (RBD) is the main region that can interact directly with ACE2, with few residues contained in Receptor Binding Motif (RBM) inside of RBD (Shang et al., 2020). Some key residues in RBM such as Leu 455, Phe 456, Ser 459, Gln 474, Ala 475, Phe 486, Phe 490, Gln 493, and Pro 499 turn as key residues to bind with ACE2 (Yi et al., 2020). Even though all of the analyzed active compounds weren't bind with those residues (Tab. 3 ), the presence of those compounds could interfere binding form of S-ACE2 (Fig. 3). Additionally, some key residues of S-ACE2 binding complex weren't find after $S$ protein bound with the GRO, SGO, ZBR, ZBO, or ZGR (supplementary table 1). This was designate that GRO, SGO, ZBR, ZBO, and ZGR have a possibility to prevent SARS-CoV2 infection.

In conclusion, GGR, GGO, GRO, SGO, ZBR, ZBO, and ZGR have good oral bioavailability and flexibility according to drug-likeness prediction using SwissADME. Among seven analyzed compounds, GGO, GRO, SGO, $\mathrm{ZBR}, \mathrm{ZBO}$, and ZGR are the best candidates which could interact with key residues in MPro, while GRO, SGO, ZBR, ZBO, and ZGR could interfere S-ACE2 complex formation, suggesting their potential as antiviral substances.

\section{Acknowlegment}

Thank you very much for the assistance of laboratory assistants from the Biocomputation Laboratory, Department of Biology, Brawijaya University. Afterward, we are also very grateful to our lecturer Prof. Widodo, Ph.D.Med.Sc., Nia Kurniawan, S.Si, MP, D.Sc., and Yoga Dwi Jatmiko, S.Sc., M.App.Sc., Ph.D. for all their advice.

\section{References}

Bzówka, M., Mitusińska, K., Raczyńska, A., Samol, A., Tuszyński, J. A., \& Góra, A. (2020). Structural and Evolutionary Analysis Indicate That the SARS-CoV-2 Mpro Is a Challenging Target for Small-Molecule Inhibitor Design. International Journal of Molecular Sciences, 21(9). doi:10.3390/ijms21093099

Chagas, C. M., Moss, S., \& Alisaraie, L. (2018). Drug metabolites and their effects on the development of adverse reactions: Revisiting Lipinski's Rule of Five. International Journal of Pharmaceutics, 549(1-2), doi:10.1016/j.ijpharm.2018.07.046

Cheng, B., \& Li, T. (2020). Discovery of alliin as a putative inhibitor of the main protease of SARS-CoV-2 by molecular docking. Biotechniques, 69, 00-00. doi:10.2144/btn-2020-0038

Dai, W., Zhang, B., Su, H., Li, J., Zhao, Y., Xie, X., Jin, Z., Liu, F., Li, C., Li, Y., Bai, F., Wang, H., Cheng, X., Cen, X., Hu, S., Yang, X., Wang, J., Liu, X., Xiao, G., ... Liu, H. (2020). Structurebased design of antiviral drug candidates targeting the SARSCoV-2 main protease. Science (New York, N.Y.). doi:10.1126/science.abb4489

Daina, A., Michielin, O., \& Zoete, V. (2017). SwissADME: A free web tool to evaluate pharmacokinetics, drug-likeness and medicinal chemistry friendliness of small molecules. Scientific Reports, 7 , 42717. doi:10.1038/srep42717

Dallakyan, S., \& Olson, A. J. (2015). Small-molecule library screening by docking with PyRx. Methods in Molecular Biology (Clifton, N.J.), 1263, 243-250. doi:10.1007/978-1-4939-2269-7_19

Dassault Systèmes BIOVIA, Discovery studio modeling environment, Version 4.5, San Diego: Dassault Systèmes (2015)

Gurung, A. B., Ali, M. A., Lee, J., Farah, M. A., \& Al-Anazi, K. M. (2020). Unravelling lead antiviral phytochemicals for the inhibition of SARS-CoV-2 Mpro enzyme through in silico approach. Life Sciences, 255, 117831. doi:10.1016/j.lfs.2020.117831

Hilgenfeld, R. (2014). From SARS to MERS: Crystallographic studies on coronaviral proteases enable antiviral drug design. The FEBS Journal, 281(18), 4085-4096. doi:10.1111/febs.12936

Islam, M. T., Sarkar, C., El-Kersh, D. M., Jamaddar, S., Uddin, S. J., Shilpi, J. A., \& Mubarak, M. S. (2020). Natural products and their derivatives against coronavirus: A review of the nonclinical and pre-clinical data. Phytotherapy Research: PTR. doi:10.1002/ptr.6700

Joshi, R. S., Jagdale, S. S., Bansode, S. B., Shankar, S. S., Tellis, M. B., Pandya, V. K., Chugh, A., Giri, A. P., \& Kulkarni, M. J. (2020). Discovery of potential multi-target-directed ligands by targeting host-specific SARS-CoV-2 structurally conserved main protease. Journal of Biomolecular Structure \& Dynamics, 1-16. doi:10.1080/07391102.2020.1760137

Li, S.-Y., Chen, C., Zhang, H.-Q., Guo, H.-Y., Wang, H., Wang, L., Zhang, X., Hua, S.-N., Yu, J., Xiao, P.-G., Li, R.-S., \& Tan, X. (2005). Identification of natural compounds with antiviral activities against SARS-associated coronavirus. Antiviral Research, 67(1), 18-23. doi:10.1016/j.antiviral.2005.02.007

Lipinski, C. A. (2004). Lead- and drug-like compounds: The rule-of-five revolution. Drug Discovery Today. Technologies, 1(4), 337341. doi:10.1016/j.ddtec.2004.11.007

Macindoe, G., Mavridis, L., Venkatraman, V., Devignes, M.-D., \& Ritchie, D. W. (2010). HexServer: An FFT-based protein docking server powered by graphics processors. Nucleic Acids Research, 38(Web Server issue), W445-449. doi:10.1093/nar/gkq311 
Mao, Q.-Q., Xu, X.-Y., Cao, S.-Y., Gan, R.-Y., Corke, H., Beta, T., \& Li, H.-B. (2019). Bioactive Compounds and Bioactivities of Ginger (Zingiber officinale Roscoe). Foods, 8(6). doi:10.3390/foods 8060185

Rasool, A., Khan, M.-U.-R., Ali, M. A., Anjum, A. A., Ahmed, I., Aslam, A., Mustafa, G., Masood, S., Ali, M. A., \& Nawaz, M. (2017). Anti-avian influenza virus H9N2 activity of aqueous extracts of Zingiber officinalis (Ginger) and Allium sativum (Garlic) in chick embryos. Pakistan Journal of Pharmaceutical Sciences, 30(4), 1341-1344.

Shang, J., Ye, G., Shi, K., Wan, Y., Luo, C., Aihara, H., Geng, Q. Auerbach, A., \& Li, F. (2020). Structural basis of receptor recognition by SARS-CoV-2. Nature, 581(7807), 221-224. doi:10.1038/s41586-020-2179-y

Tu, Y.-F., Chien, C.-S., Yarmishyn, A. A., Lin, Y.-Y., Luo, Y.-H., Lin, Y.-T., Lai, W.-Y., Yang, D.-M., Chou, S.-J., Yang, Y.-P. Wang, M.-L., \& Chiou, S.-H. (2020). A Review of SARS-CoV2 and the Ongoing Clinical Trials. International Journal of Molecular Sciences, 21(7). doi:10.3390/ijms21072657

Walls, A. C., Park, Y.-J., Tortorici, M. A., Wall, A., McGuire, A. T., \& Veesler, D. (2020). Structure, Function, and Antigenicity of the SARS-CoV-2 Spike Glycoprotein. Cell, 181(2), 281-292.e6. doi:10.1016/j.cell.2020.02.058
Wang, L., Wang, Y., Ye, D., \& Liu, Q. (2020). Review of the 2019 novel coronavirus (SARS-CoV-2) based on current evidence. International Journal of Antimicrobial Agents, 105948 doi:10.1016/j.ijantimicag.2020.105948

Xia, S., Liu, M., Wang, C., Xu, W., Lan, Q., Feng, S., Qi, F., Bao, L., Du, L., Liu, S., Qin, C., Sun, F., Shi, Z., Zhu, Y., Jiang, S., \& Lu, L. (2020). Inhibition of SARS-CoV-2 (previously 2019 $\mathrm{nCoV}$ ) infection by a highly potent pan-coronavirus fusion inhibitor targeting its spike protein that harbors a high capacity to mediate membrane fusion. Cell Research, 30(4), 343-355. doi:10.1038/s41422-020-0305-X

Yi, C., Sun, X., Ye, J., Ding, L., Liu, M., Yang, Z., Lu, X., Zhang, Y., Ma, L., Gu, W., Qu, A., Xu, J., Shi, Z., Ling, Z., \& Sun, B. (2020). Key residues of the receptor binding motif in the spike protein of SARS-CoV-2 that interact with ACE2 and neutralizing antibodies. Cellular \& Molecular Immunology, 17(6), 621-630. doi:10.1038/s41423-020-0458-Z

Zhang, L., Lin, D., Sun, X., Curth, U., Drosten, C., Sauerhering, L., Becker, S., Rox, K., \& Hilgenfeld, R. (2020). Crystal structure of SARS-CoV-2 main protease provides a basis for design of improved $\alpha$-ketoamide inhibitors. Science (New York, N.Y.), 368(6489), 409-412. doi:10.1126/science.abb3405 